\title{
Skin temperature changes during muscular static stretching exercise
}

\author{
Ulysses Franco de Oliveira', Laís Caldas de Araújo', Palloma Rodrigues de Andrade', Heleodório Honorato dos Santos', \\ Danilo Gomes Moreira ${ }^{2,3}$, Manuel Sillero-Quintana ${ }^{3}$, José Jamacy de Almeida Ferreira,** \\ 'Department of Physical Therapy, Federal University of Paraíba, João Pessoa, Brazil \\ ${ }^{2}$ Federal Institute for Education, Sciences and Technology of Minas Gerais, Campus Governador Valadares, Governador Valadares, Brazil \\ ${ }^{3}$ Faculty of Physical Activity and Sport Sciences, Universidad Politécnica de Madrid, Madrid, Spain
}

This study aimed to investigate the acute effects of stretching exercise on skin temperature, flexibility, passive muscle stiffness, and pain during hamstring stretching in healthy young subjects. Thirty-one participants $(n=31)$ were randomly divided into 2 groups: an experimental group ( $E G$, $n=15)$, which was submitted to hamstring stretching exercise for 180 sec, and the control group $(n=16)$, which was not exercised. Skin temperature (TsK) was collected in subjects' posterior thigh using a thermographic camera at 7 time points with intervals of $30 \mathrm{sec}$ each: TO (rest), T30, T60, T90, T120, T150, and T180. In addition, the knee range of motion, passive stiffness and muscle soreness (visual analogue scale) were recorded during the stretching procedure at the same times. The EG presented a small but progressive increase in TsK $\left(30.8^{\circ} \mathrm{C} \pm 0.6^{\circ} \mathrm{C}\right.$ to $\left.31.1^{\circ} \mathrm{C} \pm 0.6^{\circ} \mathrm{C}, P<0.01\right)$, with an increased range of motion $(P<0.01)$ and muscle soreness levels $(P<0.01)$ during the gradual hamstring stretching protocol. Additionally, there was an increment of passive stiffness $(P<0.01)$ that was partially reversed toward the end of the stretching period. This research presents evidence that constant, passive and progressive stretching of the hamstrings progressively increased the local TsK by about $0.3^{\circ} \mathrm{C}$ after $180 \mathrm{sec}$ of stretching. These findings may help to understand the effects of repeated stress on the muscle-tendon unit and the role of muscle stretching in warm-up protocols and rehabilitation.

Keywords: Thermography, Muscle lengthening, Temperature

\section{INTRODUCTION}

Muscle-tendon flexibility deficit is a factor for muscular injuries in humans, especially in hamstring muscles which usually suffer shortening from a sedentary lifestyle (Bishop et al., 2004). It has been shown that subjects with shortened muscles present functional deficit and they could experience difficulty in performing functional daily life activities (Batista et al., 2009). Additionally, hamstring flexibility has been reported as an important component in postural balance, injury prevention and performance of activities which demand wide knee and hip range of motion (ROM) (Schuback et al., 2004).

Muscle-tendon shortening can be prevented and treated by performing stretching exercises, and this is often used in clinical practice of physical therapy and sports training (Magnusson et al.,
1996). The stretching exercise presents acute and chronic effects that result in an immediate change in the elastic component of the muscle-tendon unit, or long-term structural changes such as an increase in the number of sarcomeres in series (Weppler and Magnusson, 2010).

The use of static stretching (SS) is a common part of most warm-up protocols prior to any general or specific physical activity. Some researchers have shown that the inclusion of short stretching protocols (30 to $90 \mathrm{sec}$ ) prior to a specific or general physical activity can ensure an increased range of motion and improve conditions for physical exercise without impairing performance (Murphy et al., 2010; Rosario and Foletto, 2015). Nelson and Kokkonen (2013) conducted a study in which heart rate (HR) and $\mathrm{VO}_{2}$ of young subjects were analyzed during $12 \mathrm{~min}$ of SS compared to placebo stretching, and found a significant $9 \%$ in-
${ }^{*}$ Corresponding author: Jose Jamacy de Almeida Ferreira

(iD https://orcid.org/0000-0002-4926-730X

Department of Physical Therapy, Federal University of Paraíba, João Pessoa-PB 58051-900, Brazil

Tel: +55-83-3216-7200, Fax: +55-83-3216-7200, E-mail: jamacy@gmail.com

Received: January 31, 2018 / Accepted: April 11, 2018
This is an Open Access article distributed under the terms of the Creative Commons Attribution Non-Commercial License (http://creativecommons.org/licenses/by-nc/4.0/) which permits unrestricted non-commercial use, distribution, and reproduction in any medium, provided the original work is properly cited. 
crease in $\mathrm{HR}$ and $44 \%$ in $\mathrm{VO}_{2}$ of the SS group. However the authors stated that this increase is not enough to obtain a warm-up effect.

A systematic review that analyse the relationship between stretching exercise and athletic performance indicated a decrease in dynamic and static muscle strength as an acute effect of stretching (Shrier, 2004). In this respect, Knudson and Noffal (2005) reported a decrease in muscle strength with onset after $20-40$ sec of performing SS. In this same direction, Behm and Kibele (2007) and Winchester et al. (2008) reported the negative effects of stretching exercises on jump height and sprint performance, respectively.

Reactive passive stiffness during muscle stretching can determine the degree of predisposition to injury of the muscle-tendon unit. Passive tension is described as a mechanical property of the muscle related to passive tissue resistance to deformation, and the stiffness is expressed by a change in tension per unit of change in length (Weppler and Magnusson, 2010). Passive tissue resistance decreases when muscle stretching is maintained for a period of time, as in the case of normal SS (Weppler and Magnusson, 2010). According to Murphy et al. (2010), increased hip ROM after SS protocol was not accomplished by increased passive leg extensor tension, thus suggesting decreased muscle stiffness after the stretching protocol.

Several studies have demonstrated changes in muscle strength and joint torque (Batista et al., 2009; Shrier, 2004), muscle-tendon flexibility (Bishop et al., 2004), range of motion (Murphy et al., 2010), passive stiffness (Weppler and Magnusson, 2010), and adaptations in muscle length with an increase in the number of sarcomeres in series (Weppler and Magnusson, 2010), among other physiological changes due to stretching exercises.

Additionally, some studies have studied skin temperature (TsK) in experimental protocols including stretching exercises as part of a warm-up (Adamczyk et al., 2012; Adamczyk et al., 2016), and for monitoring aerobic endurance and strength training (Fernández-Cuevas et al., 2014). However, these studies did not analyze the thermal pattern during stretching as a separate part of the study. Only a few studies in literature have discussed muscle temperature during stretching. In this sense, Clinch (1968) showed an increase in muscular heat production during SS in a study with "in vivo" preparations using frog Sartorius. Although there is a tendency in the literature to consider the association of heat increments and stretching being more beneficial for range of motion gain (Nakano et al., 2012; Robertson et al., 2005), to our knowledge no studies have analyzed the potential thermal skin changes after performing only stretching exercises in humans.

Infrared thermography (IRT) is a noncontact procedure which records TsK distribution using a thermal camera that receives and processes the infrared radiation emitted by the body's surface (Vainer, 2005). The heat exchange between the skin and environment occurs by radiation and is related to the area of the superficial vascular beds distributed in the region. As a result, TsK distribution depends both on core and ambient temperature, and on the variation in the area of infrared emitting vessels along the region where the temperature is measured (Ammer and Formenti, 2016). The temperature distribution within the region of interest (ROI) has been analyzed in a recent study comparing the average temperature of all the pixels representing the ROI (Troi), and the mean of the hottest pixels automatically selected within the ROI (Tmax). Although some differences were observed between the basal temperature distribution (at rest) and after exercise, the study indicated a substantial agreement between the two measurements (Formenti et al., 2017).

IRT has also been used to characterize the temperature pattern of the tendon tissue (Sanz-López et al., 2016), and is considered a valuable tool for research in physiology (Formenti et al., 2016; Sampaio et al., 2016) and in monitoring the thermal effects of athletic training (Fernández-Cuevas et al., 2014; Vainer, 2005). As IRT can record the thermal changes occurring in the muscle by measuring the TsK, we suggest that TsK may also be effective in detecting variations in temperature patterns which could be useful to set the critical limits of musculotendinous strain levels during stretching.

Thus, this study aims to characterize the acute effects of hamstring stretching exercise on TsK, flexibility, muscle passive stiffness, and pain in healthy young subjects. It was hypothesized that hamstring stretching exercise can change the temperature in the muscle-tendon unit.

\section{MATERIALS AND METHODS}

\section{Participants}

The sample size test for each group condition was determined in G*Power version 3.1.9.2 (Franz Faul, Kiel, Germany) (Faul et al., 2007). The following design specifications were taken into account: $\alpha=0.05 ;(1-\beta)=0.95$; effect size $f=0.25$; test family $=F$ test; and statistical test $=$ repeated measures analysis of variance (ANOVA), with in-between interaction. The estimated sample size according to these specifications was 26 subjects.

This is a controlled experimental study in which 36 volunteers 


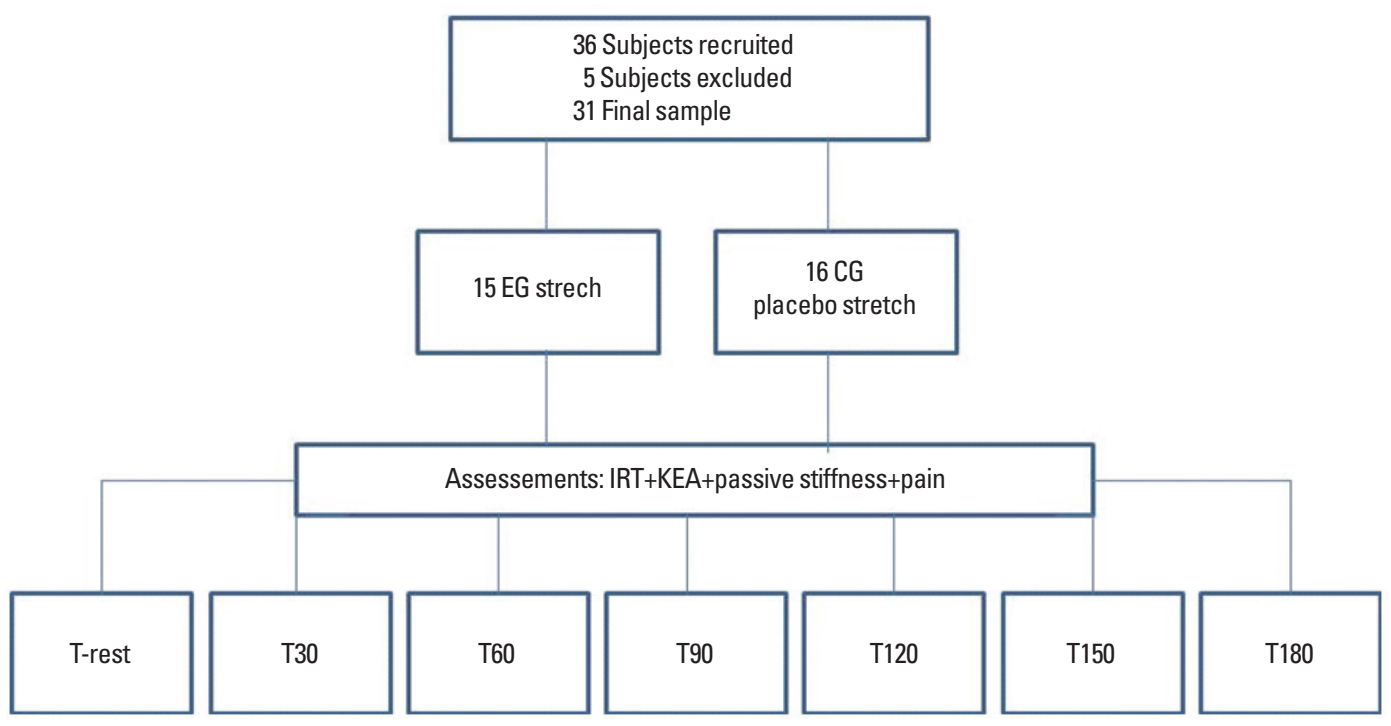

Fig. 1. Experimental design. EG, experimental group; CG, control group; IRT, infrared Thermography; KEA, knee extension angle.

were selected from both genders. Five participants were excluded after applying inclusion/exclusion criteria cited in sequence of: age between 18 and 30 years; hamstring tightness characterized by a knee extension angle of $(\mathrm{KEA}) \leq 160^{\circ}$ measured with the hip at $90^{\circ}$ of flexion; being a nonsmoker; not presenting any musculoskeletal injuries of the lower limbs or conditions that could interfere in capturing the posterior thigh temperature (Fig. 1). All subjects expressed their consent by signing an informed consent form in accordance with the rules of the Ethics Committee of the University that approved the research plan, in compliance with Resolution 466/12 of the National Health Council and the Helsinki Declaration.

Thus, the participants consisted of 31 healthy volunteers, with 15 randomly assigned to form the experimental group (EG, $n=15$, 3 males $/ 12$ females, $21.4 \pm 1.4$ years, $60.9 \pm 14.9 \mathrm{~kg}, 1.65 \pm 0.09 \mathrm{~m}$, body mass index $[\mathrm{BMI}]=22.1 \pm 3.2 \mathrm{~kg} / \mathrm{m}^{2}$ ), and 16 in the control group (CG, $\mathrm{n}=16,8$ males/ 8 females, $21.0 \pm 1.9$ years, $65.6 \pm 12.8$ $\left.\mathrm{kg}, 1.68 \pm 0.08 \mathrm{~m}, \mathrm{BMI}=23.3 \pm 3.9 \mathrm{~kg} / \mathrm{m}^{2}\right)$. Considering the dynamics of the proposed protocol, subjects classified as "physically active" according to the criteria of the American College of Sports Medicine (Garber et al., 2011) were included by having regular physical training sessions at least 3 times a week during the last 4 months.

\section{Procedures}

Each participant visited the laboratory for a preliminary session in order to perform their physical examination and hamstring flexibility test. The physical examination consisted of filling out an evaluation form containing identification, anthropometric data (age, weight, height, BMI, sex), medical history and level of physical activity.

In order to verify that the subjects met the study criteria $\left(\mathrm{KEA} \leq 160^{\circ}\right)$, we conducted the KEA test using a manual goniometer (CARCI, São Paulo, Brazil). Participants were positioned on a stretcher in the supine position with their hip flexed to $90^{\circ}$, with the goniometer placed on the side of the knee matching the axis to the lateral epicondyle of the femur (joint axis). The fixed equipment arm was positioned on the outside of the thigh, on the line connecting the axis of the knee joint to the greater trochanter of the femur. The mobile arm was positioned on the lateral line of the leg connecting the axis of the knee joint to the lateral malleolus of the ankle. We asked the participants to perform a maximum extension of the knee and maintain the position for about $10 \mathrm{sec}$, while reading the KEA on the goniometer (Batista et al., 2009).

Participants were wearing bathing shorts or a bathing suit and remained in the orthostatic position at rest for $15 \mathrm{~min}$ in the examination room to acclimatize the exposed posterior thigh (Marins et al., 2014a). The room was equipped with an air conditioner and the temperature was set to $23^{\circ} \mathrm{C} \pm 1^{\circ} \mathrm{C}$ with relative humidity below $50 \%$ for all procedures. Next, they were positioned on a stretcher in the supine position with the hip flexed to $90^{\circ}$ and stabilized with a specially-customized wooden support.

Initial knee angle was defined from a passive extension, as per- 


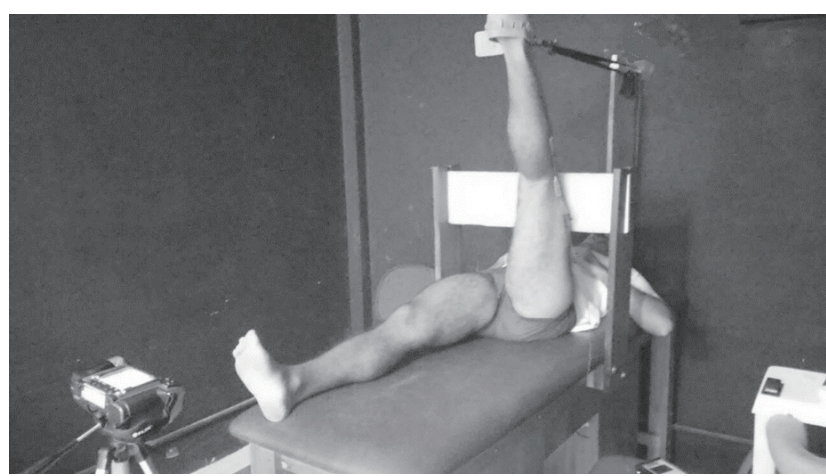

Fig. 2. Position for stretching and performing the assessments.

ceived based on the participant's first stretching; the stretching procedure was monitored throughout its duration by electrogoniometry; the angle was fixed by means of a fixing belt on the wall and onto the participants' ankle, connected in series with an isometric dynamometer, as shown in Fig. 2. Passive stiffness was recorded and gradually increased by $10 \%$ every 30 sec during stretching. Recording was done every $30 \mathrm{sec}$ by means of a digital isometric dynamometer DD-300 (Instrutherm, São Paulo, Brazil); stretching lasted 3 min, totaling a record of 7 time points with intervals of 30 sec each, being: T0 (rest), T30, T60, T90, T120, $\mathrm{T} 150$, and T180. In the event that a participant could not afford any additional adjustments due to discomfort caused by the stretching, the tension was maintained at this level, but all variables continued to be recorded until the stretching time was completed. GC was measured in the same position as EG at all times, with the limb at rest (placebo stretch) and supported by the fixing belt on the wall and on the participants' ankle, connected in series with the dynamometer, and always trying to keep the knee at the initial angle. Next, the CG participants were not submitted to any stretching during the procedure. This procedure was randomly performed for the dominant and nondominant limbs of both groups.

We used an electronic goniometer model W4X8 (Biometrics Ltd., Caerphilly, UK) to measure knee range of motion during the stretching procedure (see Fig. 2 in the lateral area of the knee). The data collected during the acute stretching assessment was expressed by an amplitude graphic corresponding to the time points T-rest to T180, from which maximum amplitude (knee angle in rad) value was extracted at each time using software DataLog (Biometrics Ltd., Caerphilly, UK). The sampling rate used in the signal capture was while excitation 1,000 Hz, output was 2,000 mV.

A visual analogue scale (VAS) was used to assess subjective leg
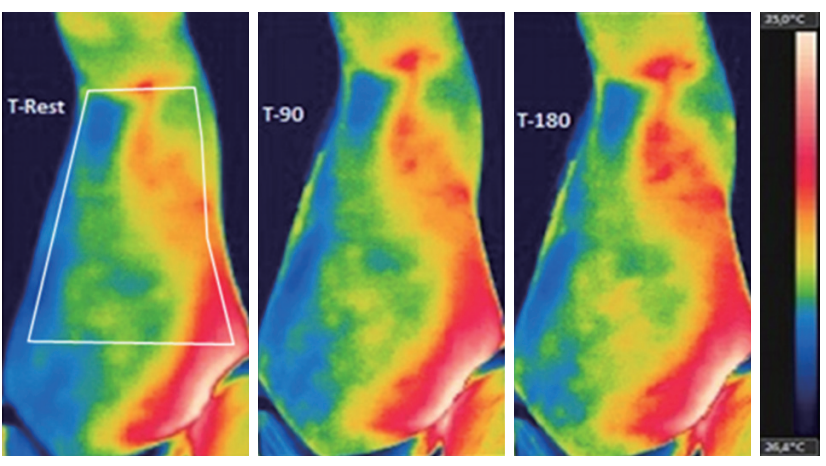

Fig. 3. Stretching sequence at rest, after $90 \mathrm{sec}$ and $180 \mathrm{sec}$. The polygon represents the temperature measurement area.

soreness. The scale had a 10-cm line marked with "no pain" at one end (0), and "extremely sore" at the opposite end (10). Each participant was directed to indicate a point along the $10-\mathrm{cm}$ line for their response to soreness.

Volunteers were instructed in accordance with the recommendations of the European Association of Thermology (Ammer, 2008) to perform the thermographic examination. Before the evaluation, we requested that the participants: (a) not drink any alcohol, coffee or any other stimulant beverage at least $12 \mathrm{hr}$ before the test; (b) avoid sunbathing or exposure to UV-rays $24 \mathrm{hr}$ before the test, (c) avoid high-intensity or exhaustive exercise at least $24 \mathrm{hr}$ before the test; (d) refrain from heavy meals $2 \mathrm{hr}$ before the test; and (e) avoid applying any moisturizing creams or any similar product on the region that would be evaluated.

For evaluating the subject's posterior thigh temperature, we used a model T-360 thermographic camera (FLIR Systems, Wilsonville, OR, USA) which operates at a frame rate of $30 \mathrm{~Hz}$, presents thermal sensitivity of $0.05^{\circ} \mathrm{C}$ with an infrared resolution of $320 \times 240$ pixels and a spectral range of $7-13 \mu \mathrm{m}$. The camera captures images of objects with temperatures between $-20^{\circ} \mathrm{C}$ to $120^{\circ} \mathrm{C}$, and has an accuracy of $\pm 2 \%$. Ambient temperature and relative humidity were recorded with a digital thermo-hygrometer (INSTRUTEMP, São Paulo, Brazil) before each measurement. The camera was positioned $1.5 \mathrm{~m}$ away from the posterior thigh region by means of a tripod, and the thermal images were taken perpendicular to the ROI (Fig. 2).

Analysis of the thermographic images was performed using thermographic software Thermacam Reporter version 8.2 (FLIR Systems). Skin emissivity was set at 0.98 (Bernard et al., 2013). For this analysis, an operator selected the ROI for each time point and for each subject. The posterior thigh was defined by a polygon that included the area of all hamstring muscles, excluding the 
Table 1. TsK, KEA, PS, and VAS results in all experimental time points

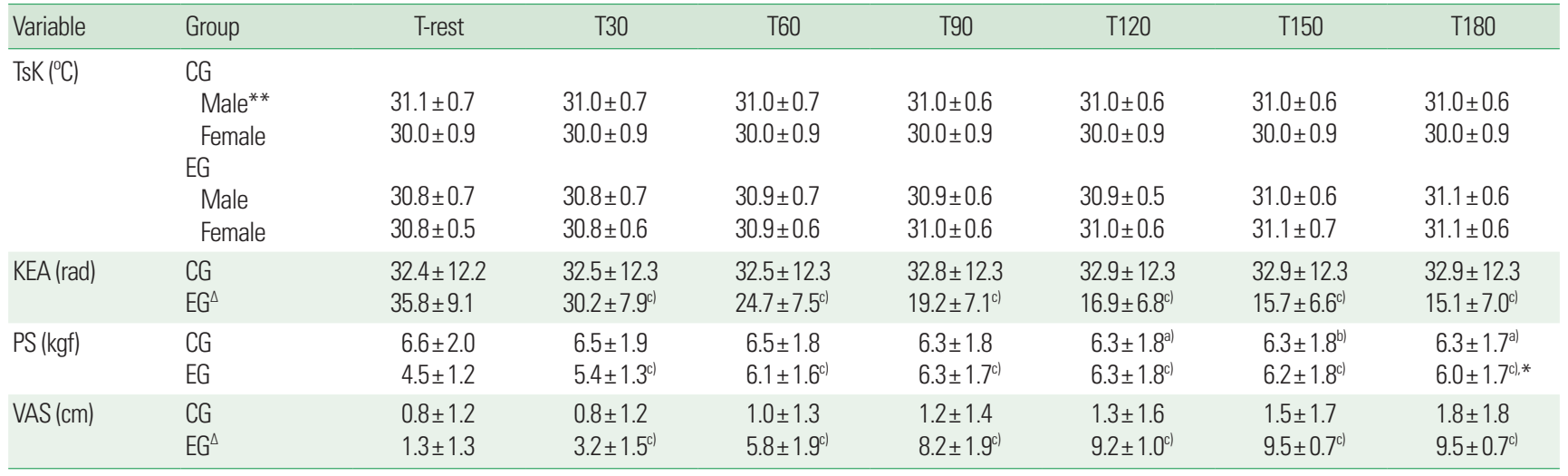

Values are presented as mean \pm standard deviation.

TsK, average skin temperature; KEA, knee extension angle; PS, passive stiffness; VAS, visual analogue scale; CG, control group; EG, experimental group. Intragroup difference from T-rest: ${ }^{\text {al }} P<0.05 .{ }^{\text {bl }} P<0.01 .{ }^{\mathrm{c})} P<0.001$.

${ }^{*} P<0.01$, intragroup difference for $T 120 .{ }^{*} P<0.05,{ }^{\Delta} P<0.01$, intergroup differences. ${ }^{* *} P<0.05$, between sex difference.

popliteal fossa. The average temperature of posterior thigh was recorded at each time point and used in all statistical procedures. Fig. 3 shows the measurement area and thermographic profile at rest, 90 sec and 180 sec during stretching.

\section{Statistical analysis}

The variables of temperature $\left({ }^{\circ} \mathrm{C}\right)$, passive stiffness (kgf), KEA $\left({ }^{\circ}\right.$ rad), and muscle soreness (VAS) were grouped and descriptive statistics were processed (mean \pm standard deviation). Data normality and homogeneity analyses were initially performed using the Kolmogorov-Smirnov and Levene test, respectively. Next, three-way ANOVA ( 2 groups $\times 2 \operatorname{sex} \times 7$ repeats) with repeated measures was applied to compare the average temperature, average passive stiffness and KEA during stretching. Bonferroni adjustment was used for the time lapse differences. A significance level of $5 \%(P<0.05)$ was considered for all analyses. Statistical analyses were performed using IBM SPSS Statistics ver. 20.0 (IBM Co., Armonk, NY, USA).

\section{RESULTS}

Overall analysis of average TsK during stretching showed that there was a significant difference when comparing the groups $F(1$, $58)=4.779, P=0.033$, times $F(2.132,123.667)=3.518, P=0.030$, sex $F(1,58)=5.701, P=0.020$, and interaction effect of times $\times$ groups $F(2.132,123.667)=8.905, P=0.001$. However, in this analysis, no interaction effect was verified for time $\times \operatorname{sex} F(2.132$, $123.667)=0.254, P=0.790$, and for time $\times$ group $\times \operatorname{sex} F(2.132$, $123.667)=0.208, P=0.826$. The temperature evolution by group

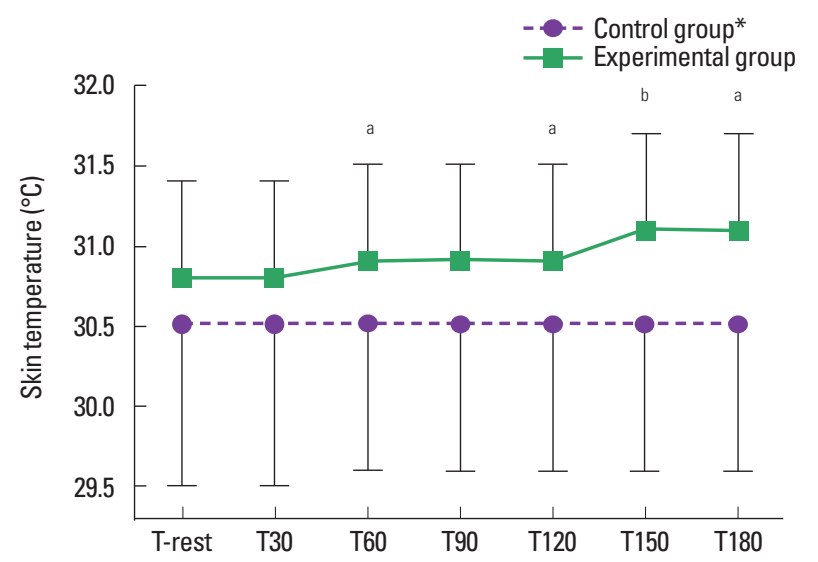

Fig. 4. Graphical representation of skin temperature during stretching in the control and experimental groups. Intragroup difference from T-rest. ${ }^{a} P<0.05$. ${ }^{\mathrm{b}} P<0.01 .{ }^{*} P<0.05$, intergroup significant difference.

and sex over time was presented in Table 1, and the Fig. 4 shows the graphical temperature evolution of the groups over time.

A significant increase in temperature was verified in the intragroup analysis for the $\mathrm{EG}$ when the times of T-rest were compared to T60 $(P=0.041)$, T120 $(P=0.021)$, T150 $(P=0.008)$, and T180 times $(P=0.001)$. The $\mathrm{CG}$ showed no significant intragroup difference $(P=0.085)$.

When analyzing the KEA evolution during stretching, significant differences were found when comparing the times $F(1.507$, $90.431)=168.041, P=0.001$, groups $F(1,60)=16.032, P=0.001$ and interaction analysis times $\times$ groups $F(1.507,90.431)=187.406$, $P=0.001$. A significant decrease in KEA was verified in the intragroup analysis considering all comparisons $(P=0.001)$, except when comparing T120 to T150 $(P=0.174)$, and T180 $(P=0.185)$ 
in the EG. The CG had no significant intragroup differences. Table 1 summarizes the results of KEA, passive stiffness and muscle soreness for the experimental and CGs.

A significant difference was found in the analysis of passive stiffness during stretching when times $F(2.246,134.760)=23.516, P=0.001$ and interaction effect of times $\times$ groups $F(2.246,134.760)=45.323$, $P=0.001$ were considered, but not for groups $(P=0.183)$. There was a significant increase of passive stiffness during stretchingin the intragroup analysis considering all comparisons with T-rest $(P=0.001)$; however, a decrease in tensile strength $(P=0.007)$ was observed for T120-T180 time comparisons in the EG. The CG showed significant intragroup decrease in tensile strength when comparing T-rest to $\mathrm{T} 120(P=0.016), \mathrm{T} 150(P=0.008)$, and T180 $(P=0.014)$ (Table 1$)$.

In the muscle soreness analysis during stretching, it was observed that there was a significant difference when comparing the times $F$ $(2.479,148.737)=179.638, P=0.001$, groups $F(1,60)=428.6389$, $P=0.001$, and interaction effect of times $\times$ groups $F(2.479,148.737)$ $=119.844, P=0.001$. In the intragroup analysis, a significant increase in pain grade was verified considering all comparisons $(P=$ 0.001), except for T120 to T150 $(P=0.118)$ and T180 times $(P=$ $0.142)$ in the EG. The CG had no significant intragroup differences (Table 1).

\section{DISCUSSION}

The results obtained in this study demonstrate a small but progressive increase in TsK with an increased range of motion and muscle soreness levels during a 180-sec protocol of gradual hamstring stretching. Additionally, there is an increment of passive stiffness that is partially reversed toward the end of the stretching period. To our knowledge this is the first study that presents TsK changes during muscle stretching in humans.

The viscoelastic conditions and muscle temperature prior to exercise have been considered an important factor in determining performance during physical activities and sports practice (Murphy et al., 2010; O'Sullivan et al., 2009; Rosario and Foletto, 2015; Samson et al., 2012). The use of warm-up protocols including general aerobic, muscle stretching and activity-specific exercises before physical activity has become a routine in athletic training and sports practice (Murphy et al., 2010; O'Sullivan et al., 2009). These protocols are designed for preparing the muscles and periarticular soft tissue to promote muscle heating and increase tissue extensibility before the main activity (Rosario and Foletto, 2015; Young, 2007; Young and Behm, 2002). In this respect, the use of stretching exercises has been controversial because some studies have shown a deleterious effect on muscle strength and power immediately after stretching (Shrier, 2004; Winchester et al., 2008). Furthermore, it was assumed that stretching does not promote muscle warming and therefore is not effective as a warm-up (Nelson and Kokkonen, 2013).

The results presented in this study show an increase in TsK in the hamstrings during $3 \mathrm{~min}$ of stretching. The increase seems small in magnitude $\left(0.27^{\circ} \mathrm{C}\right)$; however, it is significant compared to the $C G$ which reported stable temperature values.

In this study, there is a mixed sample composition of males and females. In this aspect, some studies have reported a lower temperature in several body regions of females measured at rest (Chudecka and Lubkowska, 2015; Marins et al., 2014b; Marins et al., 2015) and during aerobic exercise in a cold environment (Fournet et al., 2013) when compared to males. In this study, a temperature difference between male and female was observed, however there was no effect of the sex factor over time (Times $\times$ sex interaction). This result suggests that the sex factor did not influence TsK during stretching.

Although we have not found similar studies in the literature, some studies with horse tendons (Birch et al., 1997) and humans (Sanz-López et al., 2016) in repeated stress conditions may help to understand these results. The tendon is able to store energy, thereby improving the performance of locomotion; however, its elasticity is imperfect and a portion of this energy is dissipated as heat (Birch et al., 1997). The authors report that the central portion of the equine tendon can reach about $45^{\circ} \mathrm{C}$ in locomotion at high speeds, and that high temperature accelerates the process of tendon fiber degeneration.

Farris et al. (2011) presented a mathematical model to estimate the temperature variation in the human Achilles tendon (AT) while running. The model considers mechanical properties such as stiffness, hysteresis, and the cross-sectional area during running, and in a conservative estimation demonstrated that the temperature of the tendon reaches $41^{\circ} \mathrm{C}$.

In addition, Sanz-López et al. (2016) studied the temperature of the patellar and ATs in humans during running using IRT. The authors found an increase of about $0.7^{\circ} \mathrm{C}$ after one hour of running, except for a group adapted to eccentric exercise which had a much smaller increase $\left(0.11^{\circ} \mathrm{C}\right.$ to $\left.0.29^{\circ} \mathrm{C}\right)$. They also reported increments of $1.49^{\circ} \mathrm{C}$ and $1.21^{\circ} \mathrm{C}$ in the TsK for the right and left knees, respectively, after 40 min of aerobic exercise; values that decreased to normal after $1 \mathrm{hr}$ of recovery. At this point, it has to be considered that the knee joint is not only the patellar tendon; therefore, some of the thermogenic effects of the exercise regis- 
tered at skin level after aerobic training could not only be generated by the activity of the tendon, but also by other pro-inflammatory agents activated by the exercise.

The above mentioned findings lead us to hypothesize that kinetic energy is stored and released as heat through the skin during stretching of the muscle-tendon unit in the final part of the stretch. In this study, while the temperature reached the maximum value (T150), passive stiffness also started to decrease. There is a great possibility that these events are interconnected.

Also, passive stiffness in this study gradually increased in performing up to $150 \mathrm{sec}$ of stretching, and this time also coincides with KEA stabilization. It seems that when the tissue reached its extension threshold, its passive stiffness began to decrease. In this way, Magnusson et al. (1996) studied the mechanical properties of the hamstring muscles during stretching. The authors observed a decrease of $18 \%$ to $21 \%$ of passive torque at the end of the stretching range of motion, and concluded that this viscoelastic stress relaxation is expected when the final position of stretching is reached and maintained for a period of time. This theory is also supported in a later work by Weppler and Magnusson (2010).

The KEA measured in this study progressively decreased during stretching and stabilized at about $15^{\circ} \mathrm{rad}$ at $150 \mathrm{sec}$ of stretching time. This finding was followed by stabilization in a painful sensation at the same time. Several works report an adaptation of pain perception (referred to as "sensory theory") as a factor for achieving greater extensibility after performing stretching exercises (Magnusson et al., 1996).

A study limitation which should be pointed out is the antigravity position of the lower limb adopted during the stretching, as the thermographic skin changes reflect the heat dissipation produced in the muscles, which is then carried to the surface by way of vascularization. This position could cause a decrease in blood volume in the lower limb and, consequently, a decrease in temperature. However, this affected the exercised and control limb in the same way. It should also be considered that the stretching applied to the subjects was constant and progressive; it is possible that a fixed stretching level with recovery intervals (which are normally performed in training) could achieve different results. This point should be considered in future research.

In conclusion, our data showed that constant, passive and progressive stretching of the hamstrings progressively increases localized TsK and muscle flexibility. These findings may help to understand the effects of repeated stress on the muscle-tendon unit and the role of muscle stretching in warm-up protocols and rehabilitation.

\section{CONFLICT OF INTEREST}

No potential conflict of interest relevant to this article was reported.

\section{ACKNOWLEDGMENTS}

We appreciate the efforts of the participants during these experiments and thank them for their participation in the various experimental protocols. This research was supported by the National Council of Scientific and Technologic Development (CNPq www. cnpq.br) with the post-doctoral scholarship number 204722/20144 for JJAF and Ph.D. Scholarship number 205815/2014-6 for DGM.

\section{REFERENCES}

Adamczyk JG, Boguszewski D, Siewierski M. Physical effort ability in counter movement jump depending on the kind of warm-up and surface temperature of the quadriceps. Baltic J Health Phys Act 2012; 4:164-171.

Adamczyk JG, Olszewska M, Boguszewski D, Białoszewski D, Reaburn P. Is it possible to create a thermal model of warm-up? Monitoring of training process in athletic decathlon. Infrared Phys Technol 2016; 76:555-559.

Ammer K. The glamorgan protocol for recording and evaluation of thermal images of the human body. Thermol Int 2008;18:125-129.

Ammer K, Formenti D. Does the type of skin temperature distribution matter? Thermol Int 2016;26:51-54.

Batista LH, Vilar AC, de Almeida Ferreira JJ, Rebelatto JR, Salvini TF. Active stretching improves flexibility, joint torque, and functional mobility in older women. Am J Phys Med Rehabil 2009;88:815-822.

Behm DG, Kibele A. Effects of differing intensities of static stretching on jump performance. Eur J Appl Physiol 2007;101:587-594.

Bernard V, Staffa E, Mornstein V, Bourek A. Infrared camera assessment of skin surface temperature--effect of emissivity. Phys Med 2013; 29:583-591.

Birch HL, Wilson AM, Goodship AE. The effect of exercise-induced localised hyperthermia on tendon cell survival. J Exp Biol 1997;200(Pt 11):1703-1708.

Bishop S, Draper DO, Knight KL, Brent Feland J, Eggett D. Human tissue-temperature rise during ultrasound treatments with the aquaflex gel pad. J Athl Train 2004;39:126-131.

Chudecka M, Lubkowska A. Thermal maps of young women and men. Infrared Phys Technol 2015;69:81-87. 
Clinch NF. On the increase in rate of heat production caused by stretch in frog's skeletal muscle. J Physiol 1968;196:397-414.

Farris DJ, Trewartha G, McGuigan MP. Could intra-tendinous hyperthermia during running explain chronic injury of the human Achilles tendon? J Biomech 2011;44:822-826.

Faul F, Erdfelder E, Lang AG, Buchner A. G*Power 3: a flexible statistical power analysis program for the social, behavioral, and biomedical sciences. Behav Res Methods 2007;39:175-191.

Fernández-Cuevas I, Sillero-Quintana M, Garcia-Concepcion MA, Ribot Serrano J, Gómez-Carmona PM, Bouzas Marins JC. Monitoring skin thermal response to training with infrared thermography. New Stud Athletics 2014;29:57-71.

Formenti D, Ludwig N, Rossi A, Trecroci A, Alberti G, Gargano M, Meria A, Ammer K, Caumo A. Skin temperature evaluation by infrared thermography: comparison of two image analysis methods during the nonsteady state induced by physical exercise. Infrared Phys Technol 2017;81:32-40.

Formenti D, Ludwig N, Trecroci A, Gargano M, Michielon G, Caumo A, Alberti G. Dynamics of thermographic skin temperature response during squat exercise at two different speeds. J Therm Biol 2016;59:5863.

Fournet D, Ross L, Voelcker T, Redortier B, Havenith G. Body mapping of thermoregulatory and perceptual responses of males and females running in the cold. J Therm Biol 2013;38,339-344.

Garber CE, Blissmer B, Deschenes MR, Franklin BA, Lamonte MJ, Lee IM, Nieman DC, Swain DP; American College of Sports Medicine. American College of Sports Medicine position stand. Quantity and quality of exercise for developing and maintaining cardiorespiratory, musculoskeletal, and neuromotor fitness in apparently healthy adults: guidance for prescribing exercise. Med Sci Sports Exerc 2011;43:13341359.

Knudson D, Noffal G. Time course of stretch-induced isometric strength deficits. Eur J Appl Physiol 2005;94:348-351.

Magnusson SP, Simonsen EB, Aagaard P, Dyhre-Poulsen P, McHugh MP, Kjaer M. Mechanical and physical responses to stretching with and without preisometric contraction in human skeletal muscle. Arch Phys Med Rehabil 1996;77:373-378.

Marins JC, Fernandes AA, Cano SP, Moreira DG, da Silva FS, Costa CM, Fernandez-Cuevas I, Sillero-Quintana M. Thermal body patterns for healthy Brazilian adults (male and female). J Therm Biol 2014a;42:1-8.

Marins JC, Formenti D, Costa CM, Fernandes AA, Sillero-Quintana M. Circadian and gender differences in skin temperature in militaries by thermography. Infrared Phys Technol 2015;71:322-328.

Marins JC, Moreira DG, Cano SP, Sillero-Quintana M, Soares DD, Fernandes AA, da Silva FS, Costa CM, Soares DD, dos Santos Amorim
PR. Time required to stabilize thermographic images at rest. Infrared Phys Technol 2014b;65:30-35.

Murphy JR, Di Santo MC, Alkanani T, Behm DG. Aerobic activity before and following short-duration static stretching improves range of motion and performance vs. a traditional warm-up. Appl Physiol Nutr Metab 2010;35:679-690.

Nakano J, Yamabayashi C, Scott A, Reid WD. The effect of heat applied with stretch to increase range of motion: a systematic review. Phys Ther Sport 2012;13:180-188.

Nelson AG, Kokkonen J. Elevated metabolic rate during passive stretching is not a sufficient aerobic warm-up. J Sport Health Sci 2013;2:109114.

O'Sullivan K, Murray E, Sainsbury D. The effect of warm-up, static stretching and dynamic stretching on hamstring flexibility in previously injured subjects. BMC Musculoskelet Disord 2009;10:37.

Robertson VJ, Ward AR, Jung P. The effect of heat on tissue extensibility: a comparison of deep and superficial heating. Arch Phys Med Rehabil 2005;86:819-825.

Rosario JL, Foletto Á. Comparative study of stretching modalities in healthy women: heating and application time. J Bodyw Mov Ther 2015;19:3-7.

Sampaio L, Bezerra E, Paladino K, dos Santos JO, Priego-Quesada JI, Rossato $\mathrm{M}$. Effect of training level and blood flow restriction on thermal parameters: preliminary study. Infrared Phys Technol 2016;79:25-31.

Samson M, Button DC, Chaouachi A, Behm DG. Effects of dynamic and static stretching within general and activity specific warm-up protocols. J Sports Sci Med 2012;11:279-285.

Sanz-López F, Martínez-Amat A, Hita-Contreras F, Valero-Campo C, Berzosa $\mathrm{C}$. Thermographic assessment of eccentric overload training within three days of a running session. J Strength Cond Res 2016;30: 504-511.

Schuback B, Hooper J, Salisbury L. A comparison of a self-stretch incorporating proprioceptive neuromuscular facilitation components and a therapist-applied PNF-technique on hamstring flexibility. Physiotherapy 2004;90:151-157.

Shrier I. Does stretching improve performance? A systematic and critical review of the literature. Clin J Sport Med 2004;14:267-273.

Vainer BG. FPA-based infrared thermography as applied to the study of cutaneous perspiration and stimulated vascular response in humans. Phys Med Biol 2005;50:R63-94.

Weppler $\mathrm{CH}$, Magnusson SP. Increasing muscle extensibility: a matter of increasing length or modifying sensation? Phys Ther 2010;90:438-449.

Winchester JB, Nelson AG, Landin D, Young MA, Schexnayder IC. Static stretching impairs sprint performance in collegiate track and field athletes. J Strength Cond Res 2008;22:13-19. 
Young WB. The use of static stretching in warm-up for training and competition. Int J Sports Physiol Perform 2007;2:212-216.
Young WB, Behm DG. Should static stretching be used during a warmup for strength and power activities? Strength Cond J 2002;24:33-37. 